\title{
Monitoring and analysis of anomalous refraction using a digital zenith camera system
}

\author{
C. Hirt \\ Institut für Erdmessung, Universität Hannover, Schneiderberg 50, 30167 Hannover, Germany \\ e-mail: Chris.Hirt (at)web. de \\ Received 24 April 2006 / Accepted 1 August 2006
}

ABSTRACT

\begin{abstract}
Context. Anomalous refraction is considered to be a limiting factor for ground-based astrometry in general and astrogeodetic observations in particular. Typical characteristics of anomalous refraction are basically known by means of spot-check-data, however the fluctuation is rather little studied.

Aims. The goal of this study is to derive empirical knowledge on the fluctuation of anomalous refraction in the optical domain.

Methods. A Digital Zenith Camera System usually applied for the astrogeodetic determination of the Earth's gravity field has been used for continuously monitoring anomalous refraction. With a sample frequency of about 2 observations per minute, about 7300 single observation epochs were collected during 6 nights. Residuals of the observations with respect to reference data show how anomalous refraction behaves.

Results. The analysis of approximately $70 \mathrm{~h}$ of observational data reveals heterogeneous fluctuation patterns of anomalous refraction at the zenith. Wave-like and bump-like variations appear as well as slowly-changing, drift-like structures. With respect to its magnitude, the effect reaches from $0 . ' 05$ up to about 0.2 at frequencies of some hours. Even much lower frequencies of anomalous refraction are indicated in the data sets causing an offset of about $0{ }^{\prime} 04$. The accuracy of the filtered data has been found to be about $0{ }^{\prime} 05-0{ }^{\prime} 08$. Conclusions. The study indicates how anomalous refraction may fluctuate. The results are considered to give an estimate of the accuracy limit for astrogeodetic and other absolute ground-based astrometric observations.
\end{abstract}

Key words. astrometry

\section{Introduction}

Ground-based astrometric observations are significantly affected by the influence of astronomical refraction. The phenomenon of astronomical refraction may be subdivided into a radial-symmetric and an asymmetric component. The theory of radial-symmetric refraction presumes a spherically symmetric density distribution of the atmosphere (e.g. Teleki 1979). Radialsymmetric refraction, often also referred to as pure or normal refraction, merely depends on the zenith distance of the star, however not on its azimuth. This well-known effect disappears completely at the zenith and reaches its maximum near the horizon where stars are apparently raised to about $30^{\prime}$. Traditionally, refraction tables have been used for the computation of radialsymmetric refraction (e.g. tables from Radau, Harzer, Bessel). Today, refined and very precise models are available for computing the radial-symmetric portion of astronomical refraction directly (e.g. Stone 1996; Wittmann 1997; Auer \& Standish 2000).

Asymmetric refraction, often named as anomalous refraction (AR), is that part of astronomical refraction which cannot be explained by radial-symmetric refraction models. Hence, AR may be defined as the difference between true refraction and radial-symmetric refraction (Teleki 1979). Contrary to radialsymmetric refraction, the influence of $A R$ is a function both of zenith distance and azimuth of the star. As a consequence, AR may even refract stars at the zenith.

\subsection{Anomalous refraction and astrometry}

For ground-based astrometry, AR plays an important role as it causes additional angular displacements of observed stars.
Relative ground-based astrometric surveys, where the positions of new objects (stars, minor planets) are interpolated into a field of reference stars, do not suffer in the same way as absolute astrometry. In relative applications, AR is widely absorbed by the transformation parameters - apart from a minor differential portion of AR which may remain in the data. Absolute astrometric results, however, are strongly influenced as AR fully propagates into the astrometric results and deteriorates the accuracy considerably. Earlier examples of this absolute kind of astrometry are observations obtained with Photographic Zenith Tubes (PZT) for the determination of Earth's orientation in space (variation of Universal Time and polar motion), see e.g. Nakajima (1979) or Vondrák et al. (1995). Also absolute ground-based astrometry of early fundamental catalogues (e.g. FK4) or meridian circle observations performed in the pre-Hipparcos-era are subject to AR (Hughes 1979; Stone et al. 1996). A present example where AR plays an essential role is found in geodetic astronomy. Here, absolute astrometric observations are carried out with zenith cameras in order to determine the direction of the plumb line (e.g. Hirt 2003). The accuracy of such astrogeodetic measurements is affected and limited by the presence of AR.

\subsection{Sources of anomalous refraction}

AR most likely results from tilted atmospheric layers of equal density with respect to the plumb line of the observation site (Ramsayer 1970; Kovalevsky 1998). The tilting of atmospheric layers is the consequence of pressure and temperature gradients occurring in the horizontal direction. A classification of possible sources of $\mathrm{AR}$ in regional and local domains seems to be 
useful: regionally, changing weather situations (e.g. moving high-pressure weather fronts) may result in tilted atmospheric layers due to the associated horizontal pressure-gradients. Moreover, the regional distribution of surface density and temperature may cause a season-dependent tilt of atmospheric layers with resulting AR of a few 0.'01 (Sugawa \& Kikuchi 1979).

On the other hand, AR may also appear locally as a consequence of a particular environment of the observation site. Illustrative examples are local horizontal temperature gradients at land-sea-transitions or over strongly inclined terrain where the lower part of the atmosphere is following the surface. The latter situation may cause amplitudes of AR of 0 .' 1 or even more according to Bretterbauer (1965). Also urban heat islands have been discussed as possible local sources for tilted atmospheric layers and AR (Hughes 1979; Currie 1979).

\subsection{This study and previous works}

The phenomenon of anomalous refraction has been theoretically treated by different authors, e.g. Schubart (1954); Ramsayer (1970); Dimopoulos (1982). Empirical studies in the past focused on the indirect observation of AR by means of meteorological data (e.g. radio sonde data) which in turn were used for the computation of horizontal gradients, tilt angles and AR amplitudes (e.g. Sugawa 1956, 1958). Studies dealing with the direct observation of AR have been carried out in the past e.g. by Ramsayer (1967); Nakajima (1979); Dimopoulos (1982). The PZT-data analysed by Nakajima \& Dimopoulos as well as astrolabe-data studied by Ramsayer provide spot-check-like information on AR because just a few observations per night have been performed. The conclusion of the three studies is that amplitudes of AR vary from a few 0 .' 01 to about 0 .'2. Unfortunately, the presented results had been influenced inevitably by significant errors of star catalogues from the pre-Hipparcos-era which is why AR tended to be rather overestimated. Further studies were carried out at the USNO Flagstaff station using meridian circle observations with digital image sensors. Stone et al. (1996) detected AR at time scales of 3-40 min and inferred that AR affects observations in zenith direction by about 0. .'09. Pier et al. (2003) later showed that AR is spatially coherent at scales of at least $2^{\circ}$. However, the fluctuation of AR - the time-dependent variation of amplitudes during the night - is still less investigated, especially at larger time scales of some hours.

The objective of the work presented in this paper is to analyse the fluctuations of anomalous refraction directly by means of a continuous series of high-frequency observations in zenith direction. The study is performed in the optical domain and focuses on the mid- and low-frequency portion of AR, ranging from about $20 \mathrm{~min}$ to several hours. High-frequency refraction anomalies (several minutes) as well as very high-frequency refraction anomalies, known as (direction) scintillation, are not treated by this study.

Scintillation has been extensively studied in the past, e.g. by Han \& Gatewood (1995); Christian \& Racine (1985); Høg (1968). For a comprehensive study of anomalous refraction at radio wavelengths, the reader is referred to Olmi (2001).

\subsection{Instrumentation}

The telescope used for this study of AR is the Digital Zenith Camera System TZK2-D, a transportable field instrument constructed and operated by the University of Hannover (e.g. Hirt 2004; Hirt \& Bürki 2002). Usually, this high-precision sensor is
Table 1. Specifications of the Digital Zenith Camera System TZK2-D.

\begin{tabular}{ll}
\hline \hline Lens & Carl Zeiss Mirotar \\
Aperture & $200 \mathrm{~mm}$ \\
Focal length & $1020 \mathrm{~mm}$ \\
CCD camera & Apogee KX2E \\
Image sensor & Kodak KAF 1602E \\
Pixel count & 1.56 million \\
Pixel size & $9 \mu \mathrm{m} \times 9 \mu \mathrm{m}$ \\
Pixel scale & $1^{\prime \prime} 86 \mathrm{Pixel}^{-1}$ \\
Field of view & $0.79 \times 0.53\left(=0.42 \mathrm{deg}^{2}\right)$ \\
\hline
\end{tabular}

applied at field stations for the automatic determination of the direction of the plumb line. This astrogeodetic observable provides valuable information on the local shape of the gravity field of the Earth. The measurement system TZK2-D - like other astrogeodetic instruments - is sensitive both to the gravity field and the field of anomalous refraction. The latter is disadvantageous from a geodetic point of view. However, this sensitivity makes the zenith camera a well-suited sensor for sampling and studying $\mathrm{AR}$ in zenith direction.

The system TZK2-D consists of two major components. Firstly, a Mirotar lens, similar to Maksutov-Cassegrain, is used in combination with a CCD-camera (Apogee KX2E) for star imaging (cf. specifications listed in Table 1). The digital telescope is applied for the astrogeodetic determination of the direction of the plumb line defined by astronomical latitude and longitude $(\Phi, \Lambda)$. The second component is a GPS-receiver which is used for satellite-based timing and determination of geodetic coordinates $(\varphi, \lambda)$ of the camera. The difference of both coordinates, referred to as vertical deflections $(\xi, \eta)$, is obtained by combining both components:

$\xi=\Phi-\varphi \quad \eta=(\Lambda-\lambda) \cos \varphi$

The component $\xi$ is aligned to the local meridian whereas the component $\eta$ is oriented in the prime vertical (East-West direction). Subsequently, anomalous reflection is analysed on the basis of vertical deflections $(\xi, \eta)$ as variations are not influenced by constant geodetic coordinates $(\varphi, \lambda)$. The Digital Zenith Camera is a non-tracking and non-scanning instrument. Star images are usually taken at short exposure times (some tenths of seconds) in order to avoid trails. Due to the short exposure time and the optical specifications of the instrument, a limiting magnitude of about 13-14 mag is obtained. In comparison to similar telescopes used in tracking or scanning mode, the image data of the zenith camera TZK2-D is rather undersampled. For most of the imaged stars (9th magnitude and fainter), just a fraction (below $10 \%$ ) of the CCD's dynamic range and well capacity is exploited. Even the images of brighter stars around 7th-8th magnitude are still below the saturation level.

Before observation, the camera is set up into the plumb line (=counterpart of zenith direction) by means of high precision tilt meters. These sensors also monitor changes of the alignment during observation. Hence, instrumental tilting has no impact on the results presented in this study. The zenith camera is equipped with motorized mechanics which allows observing in two opposite camera directions. That way instrumental error sources (changes of zero offsets of CCD and tilt meters due to thermal effects or transport of the instrument) do not affect the observational results. 
Table 2. Observation data - date, time spans and number of observation epochs collected.

\begin{tabular}{lcccc}
\hline \hline Date & $\begin{array}{c}\text { Start } \\
{[\mathrm{MJD}]}\end{array}$ & $\begin{array}{c}\text { End } \\
{[\mathrm{MJD}]}\end{array}$ & $\begin{array}{c}\text { Span } \\
{[\mathrm{h}]}\end{array}$ & $\begin{array}{c}\text { Number of } \\
\text { Solutions }\end{array}$ \\
\hline 20060109 & 53744.70 & 53745.27 & 13.84 & 1419 \\
20060110 & 53745.67 & 53745.89 & 5.32 & 519 \\
20060112 & 53747.68 & 53748.20 & 12.37 & 1450 \\
20060113 & 53748.83 & 53749.27 & 10.60 & 1321 \\
20060114 & 53749.68 & 53750.25 & 13.72 & 1487 \\
20060115 & 53750.70 & 53751.24 & 12.96 & 1113 \\
\hline Total & & & 68.81 & 7309 \\
\hline
\end{tabular}

\section{Astrogeodetic observations}

The Digital Zenith Camera System TZK2-D was used for comprehensive observation of vertical deflection data $(\xi, \eta)$ during 6 nights in January 2006. The observation epochs coincide with the winter solstice in good approximation (less than one month difference). Hence, the study takes advantage of maximum night lengths allowing continuous observations during 13-14 h of darkness. Due to the winter season, the campaign took place under rather harsh environmental conditions (temperatures of about $-5^{\circ}$ Celsius). The observations were carried out at an observation site in the immediate vicinity of buildings of the University of Hannover (geodetic latitude $\varphi \approx 52^{\circ} .38$ ).

The location may be characterized as urban area with adjacent large vegetation areas (e.g. parks). Therefore it seems reasonable to assume a rather irregular, heterogeneous distribution of both ground temperature and inclination of local atmospheric layers of equal density. The observations cover 7 consecutive periods of darkness with the exception of 1.5 nights when a cloudy sky prevented taking measurements. This is seen by the observation statistics in Table 2 and by the distribution of observations in Fig. 1. During a total of about $68.8 \mathrm{~h}$ of darkness, 7309 single observation epochs were collected for analysis. A maximum value of 1487 consecutive single epochs was gained on January 14, 2006 (Table 2). The author believes that this comprehensive data set acquired and analysed in the sequence is one of the first which is able to indicate how AR fluctuates.

\section{Data processing}

For the astrometric reduction of the image data to the International Celestial Reference System ICRS, highly-precise star fields from the upcoming final version of the United States Naval Observatory CCD Astrograph Catalogue (UCAC) are used which had been kindly provided by the USNO. For the current release UCAC-2 the reader is referred to Zacharias et al. (2004). The direction of the plumb line $(\Phi, \Lambda)$ and the vertical deflection data $(\xi, \eta)$ are computed from the image and catalogue data by applying standard astrometric algorithms, which can be briefly described as follows.

For the measurement of the star coordinates, either a simple image moment analysis is performed or an analytical point spread function (typically of Gaussian type) is used for fitting the star images. For the available shortly exposed image data, there exists just a slight accuracy advantage of the point spread function over centering with image moment analysis. The single star accuracy is about $0 . ' 3$ for the point spread function and $0 ., 4$ for image moment analysis (Hirt 2004). Direction scintillation is assumed to be the limiting error source with amplitudes of some $0 . ' 1$ at exposure times of a few $0.1 \mathrm{~s}$ (see Høg 1968).
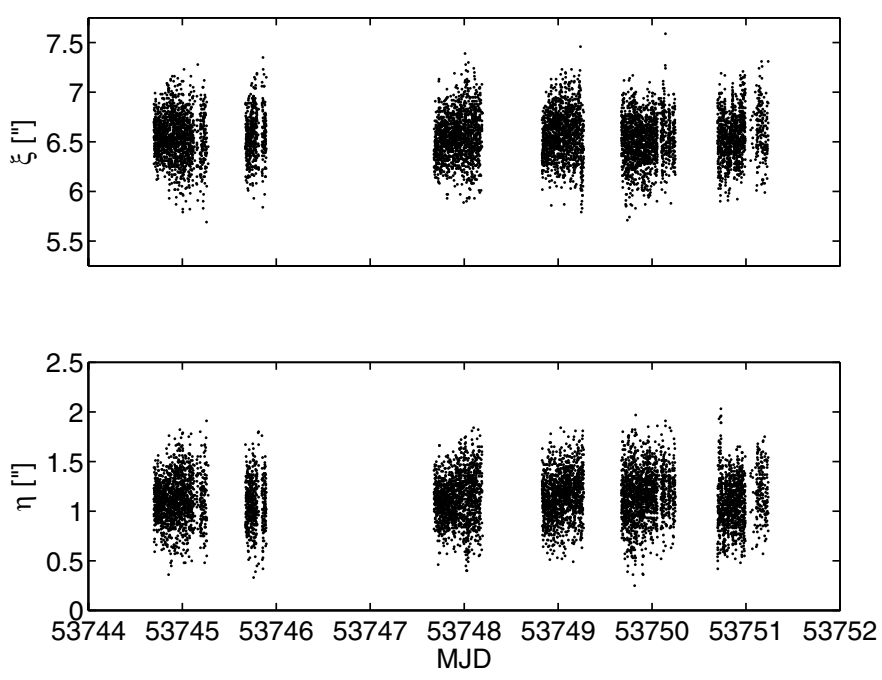

Fig. 1. Distribution of observations.

Table 3. Processed observations - number of solutions per hour (rounded), star count, number of stars used per single solution (rounded) and approximate astrometric accuracy $\sigma$ for directions to single stars. A total number of 479308 stars was processed and used for data analysis.

\begin{tabular}{lrrcc}
\hline \hline Date & $\begin{array}{r}\text { Solutions } \\
\text { per hour }\end{array}$ & $\begin{array}{r}\text { Processed } \\
\text { stars }\end{array}$ & $\begin{array}{c}\text { Stars per } \\
\text { solution }\end{array}$ & $\begin{array}{c}\text { Accuracy } \\
\sigma\left[{ }^{\prime \prime}\right]\end{array}$ \\
\hline 20060109 & 103 & 97647 & 69 & 0.41 \\
20060110 & 98 & 29600 & 57 & 0.48 \\
20060112 & 117 & 99150 & 68 & 0.39 \\
20060113 & 125 & 68047 & 52 & 0.40 \\
20060114 & 108 & 104455 & 70 & 0.39 \\
20060115 & 86 & 80409 & 72 & 0.43 \\
\hline Mean & 106 & 95862 & 66 & 0.42 \\
\hline
\end{tabular}

The centered image coordinates and their match from the UCAC star catalogue are related through a 4-parametertransformation being the sufficient transformation model for the zenith camera data. After astrometric data reduction, the zenith point is interpolated into the star field. The processing results are individually corrected for the influence of Earth orientation (sidereal time, motion of celestial and terrestrial pole) and instrumental tilt.

It is interesting to note that both methods for star image centering lead to quite similar results for the $(\Phi, \Lambda)$ and $(\xi, \eta)$ values and their standard deviations which typically amount to 0. 2-0!'3. For details on the astrometric data processing and further accuracy aspects see Hirt (2004); Hirt \& Bürki (2002).

The distribution of processed observations with respect to the epoch (Modified Julian Date, MJD) is depicted in Fig. 1. Day and night periods and the data gap of 1.5 nights are visible. Table 3 lists the observation count per hour, being a measure for the sample frequency. The average number of observations varies between 86 and 125 observations per hour. The variations result from short interruptions of the observation process due to levelling and focusing of the instrument. The resulting average sample frequency is 106 observations per hour or about 1.8 observations per minute, respectively. It is clear that very short-periodic effects, e.g. due to direction scintillation, are not addressed by this study. The last columns of Table 3 give the number of stars used for data processing (a total of about 480000 ), the star count per single solution (about 65 stars) and 
Table 4. Observation classes and their statistical characteristics (approximated values). The listed values in the last column strongly depend on the observation time due to the variable star distribution.

\begin{tabular}{lcccc}
\hline \hline $\begin{array}{l}\text { Observation } \\
\text { class }\end{array}$ & Index & Duration & $\begin{array}{c}\text { Solutions } \\
{\left[10^{3}\right]}\end{array}$ & $\begin{array}{c}\text { Stars } \\
{\left[10^{3}\right]}\end{array}$ \\
\hline Single value & - & $30 \mathrm{~s}$ & 0.001 & 0.065 \\
20 min-obs. & $20 \mathrm{~m}$ & $20 \mathrm{~m}$ & 0.051 & 3.3 \\
2 h-obs. & $2 \mathrm{~h}$ & $2 \mathrm{~h}$ & 0.201 & 13 \\
\hline Nightly mean & ngt & $11 \mathrm{~h}$ & 1.2 & 78 \\
Series mean & ser & $69 \mathrm{~h}$ & 7.3 & 480 \\
Reference & ref & $200 \mathrm{~h}$ & 20 & 1300 \\
\hline
\end{tabular}

Table 5. Results part 1 - Nightly mean values $(\xi, \eta)_{\text {ngt }}$ and deviations from the reference values $(\xi, \eta)_{\text {ref }}$. The bottom rows give the series mean $(\xi, \eta)_{\text {ser }}$, the reference values $(\xi, \eta)_{\text {ref }}$ and the remaining differences.

\begin{tabular}{lcccc}
\hline \hline Date & $\begin{array}{c}\xi_{\text {ngt }} \\
{\left[{ }^{\prime}\right]}\end{array}$ & $\begin{array}{c}\eta_{\text {ngt }} \\
{\left[{ }^{\prime}\right]}\end{array}$ & $\begin{array}{c}\xi_{\text {ngt }}-\xi_{\text {ref }} \\
{\left[{ }^{\prime \prime}\right]}\end{array}$ & $\begin{array}{c}\eta_{\text {ngt }}-\eta_{\text {ref }} \\
{\left[{ }^{\prime \prime}\right]}\end{array}$ \\
\hline 20060109 & 6.547 & 1.115 & 0.039 & 0.001 \\
20060110 & 6.560 & 1.070 & 0.052 & -0.044 \\
20060112 & 6.553 & 1.129 & 0.045 & 0.015 \\
20060113 & 6.572 & 1.153 & 0.064 & 0.039 \\
20060114 & 6.517 & 1.167 & 0.009 & 0.053 \\
20060115 & 6.560 & 1.098 & 0.052 & -0.016 \\
\hline$(\xi, \eta)_{\text {ser }}$ & 6.550 & 1.129 & 0.042 & 0.015 \\
$(\xi, \eta)_{\text {ref }}$ & 6.508 & 1.114 & & \\
\hline
\end{tabular}

the astrometric accuracy of a single star observation. A nontracking instrument like the system TZK2-D with the optical specifications described above is doing single-star astrometry typically on an accuracy level of $0 . ' 4$. However, due to the redundancy of observation, random errors are significantly reduced thus allowing to detect even small effects of AR as shown below.

\section{Data analysis}

\subsection{Observation classes}

Before analysing the data in more detail several observation classes are defined with respect to the duration or redundancy of observation, respectively. The basic observation class is the single observation $(\xi, \eta)$ resulting from a single measurement in two opposite camera directions. This procedure takes about $30 \mathrm{~s}$. During observation periods of $20 \mathrm{~min}$ ( or $2 \mathrm{~h}$, respectively), usually 50 (or 200) single measurements are carried out. The arithmetic mean values are named 20 -min-observation $(\xi, \eta)_{20 \mathrm{~m}}$ and 2-hour-observation $(\xi, \eta)_{2 \mathrm{~h}}$, respectively. Due to their reduced noise level, both observation classes play an important role for this study. Both classes are able to provide information on AR at time scales of $20 \mathrm{~min}$ and $2 \mathrm{~h}$, respectively, up to several hours - while suppressing information on AR at shorter time scales. Window widths shorter than 20 min do not typically contain enough single observations to provide mean values with sufficient accuracy and, in turn, credible information on AR.

For comparison purposes it is useful to introduce the observation classes nightly mean $(\xi, \eta)_{\text {ngt }}$ and series mean $(\xi, \eta)_{\text {ser }}$ being the mean of 7309 single observations. Finally, highlyprecise comparison values $(\xi, \eta)_{\text {ref }}$ are available at the selected observation site as the result of about 20000 single observations performed within 70 nights from 2003-2005 (currently
Table 6. Results part 2 - Standard deviations of the single observations $(\xi, \eta)$ and nightly mean values $(\xi, \eta)_{\text {ngt }}$.

\begin{tabular}{lll}
\hline \hline Standard deviation & $s_{\xi}\left[^{\prime \prime}\right]$ & $s_{\eta}\left[^{\prime \prime}\right]$ \\
\hline$(\xi, \eta)$ & 0.23 & 0.25 \\
$(\xi, \eta)_{\text {ngt }}$ & 0.019 & 0.036 \\
\hline
\end{tabular}
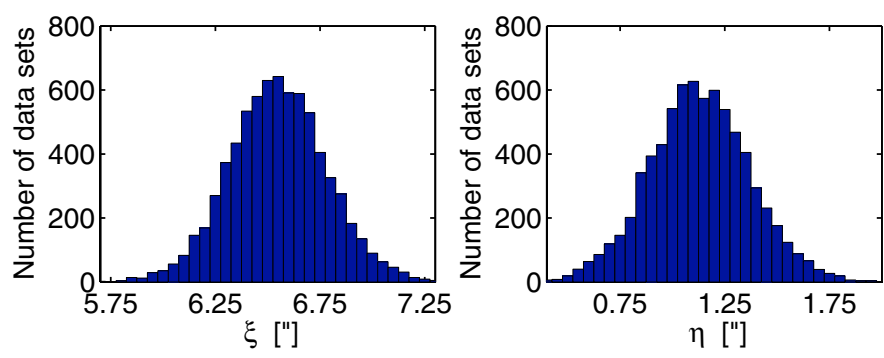

Fig. 2. Histogramm of 7309 single observations $(\xi, \eta)$ performed during the campaign. The single observations follow the normal distribution in good approximation.

unpublished). This solution relies on a total of about 1.3 million processed stars. Therefore, and due to the wide spectrum of environmental conditions (season, temperature, pressure and resulting refraction) covered by the data, it may be assumed that the reference values $(\xi, \eta)_{\text {ref }}$ are accurate to a few 0 .' $^{\prime} 01$ as AR is randomised and cancelled out for its largest part. This estimation is empirically corroborated by the spreading of mean values formed over the seasons. It should be noted that the error propagation law formally would give an extremely low standard deviation of about 2 mas for the reference values. This - clearly too optimistic - accuracy estimation differs from the above value by about one order of magnitude. The more realistic estimation of a few 0.01 takes into account that small correlations contained in the observation data prevent errors from going down indefinitely with the square root of the observations. Table 4 summarises the observation classes.

\subsection{Observation results}

The nightly mean values $(\xi, \eta)_{\text {ngt }}$ are compiled in Table 5 as well as their differences with respect to the reference values $(\xi, \eta)_{\text {ref }}$. In comparison with the highly-precise reference values $(\xi, \eta)_{\text {ref }}$, a good agreement of 0.015 is achieved for $\eta$. The value for $\xi$ which amounts to 0.042 indicates a minor systematic effect which is further discussed in Sect. 5.

Table 6 lists the standard deviation of the data sets. The accuracy of single observations has been found to be about $0.23-0 . ' 25$, corresponding well with previous data sets (Hirt 2004). The spreading and distribution of the single observation is illustrated in Fig. 2.

The very low standard deviation of about $0{ }^{\prime} 02$ and 0. '04 for the nightly mean values $(\xi, \eta)_{\text {ngt }}$ reveals an excellent repeatability of the observation of nightly mean values and a strong suppression of observational noise previously contained in the single values $(\xi, \eta)$, e.g. due to atmospheric refraction or random instrumental errors.

\subsection{Time series analysis}

In order to study the fluctuations of AR in the zenith direction, the observational data are treated as time series. A simple and 

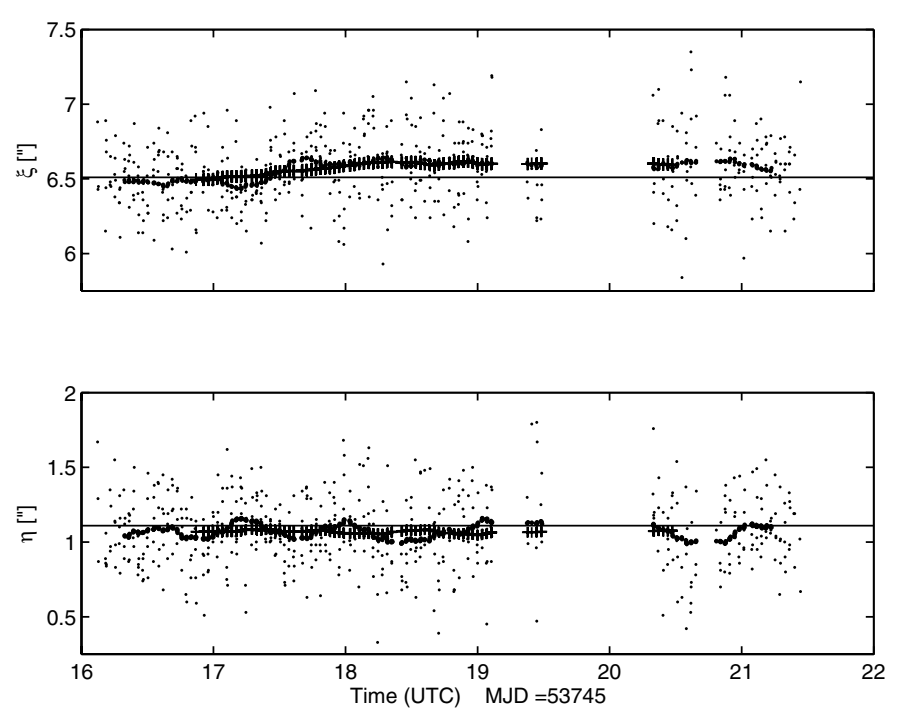

Fig. 3. Orginal observations (thin dots) and filtered observation data (20-min-data: thick dots, 2-h-data: + marker) on 10th January 2006.

efficient strategy for data smoothing is to apply a moving average filter on the single observations $\xi_{j}$ :

$\overline{\xi_{i}}=\frac{1}{\sum w_{j}} \sum_{j=i-h}^{i+h} \xi_{j} \cdot w_{j}$,

where $w$ is a weight function, e.g. a rectangular window, $h$ is the half width of the window and index $j$ runs from $i-h$ to $i+h$ for individual filtered values $\bar{\xi}_{i}$. The center of the window $i$ is moved from $h$ to $j_{\max }-h$ ( $j_{\max }=$ length of data series) in order to generate the filtered data series $\bar{\xi}$. Using a half window width $h$ of 25 values gives a smoothed series of $(\xi, \eta)_{20 \mathrm{~m}}$ where wavelengths shorter than $20 \mathrm{~min}$ are suppressed. Correspondingly, just the very long wavelengths above about $2 \mathrm{~h}$ are transferred into the $(\xi, \eta)_{2} \mathrm{~h}$-series when a filter with a half window width $h$ of 100 values is applied. That way high-frequency, random observation noise (due to instrument, scintillation) is widely eliminated from the single observations as shown in Fig. 3. Comparisons with other filter techniques (e.g. low pass filter using a cut-off frequency and a Hammingwindow) yielded similar results.

The idea behind the time series analysis is to consider residual differences between filtered data sets and reference values. Differences obtained in comparison with the nearly variance-free reference values $(\xi, \eta)_{\text {ref }}$ are likely to be caused by AR occurring above the observation site. An example is given by the data set acquired on 10th January (cf. Fig. 3). The filtered observation data $\eta_{20 \mathrm{~m}}$ and $\eta_{2 \mathrm{~h}}$ show an overall good agreement with its reference $\eta_{\text {ref }}-$ other than the filtered $\xi$-data which differ from the reference $\xi_{\text {ref }}$ by about $0{ }^{\prime} 1$ from $18 \mathrm{~h}$ to $21 \mathrm{~h}$ UTC likely due to AR.

Smoothed data sets $(\xi, \eta)_{20 \mathrm{~m}}$ and $(\xi, \eta)_{2 \mathrm{~h}}$ have been computed for the five nights (9th January 2006 and 12th till 15th January 2006) with at least ten hours of observation data available. The smoothed series, the main result of this study, are given as a function of the observation time with respect to the reference values $(\xi, \eta)_{\text {ref }}$, visualised as straight lines, in Figs. 4 a to $4 \mathrm{e}$. The $(\xi, \eta)_{20 \mathrm{~m}}$-data show wave-like structures with amplitudes varying between some 0 ' $^{\prime} 01$ and about 0 '. $^{\prime} 1$ and a frequency of about $2.8 \times 10^{-4} \mathrm{~Hz}$ (corresponding to a wavelength of about $1 \mathrm{~h}$ ). However, maximum amplitudes of up to $0 .{ }^{\prime} 3$ are visible (cf. Fig. 4c, component $\xi_{20 \mathrm{~m}}$ during the morning hours).

Due to the larger window width, the filtered $(\xi, \eta)_{2} \mathrm{~h}$ data series is much smoother than that of the 20-min-observations. The previously visible and dominating wavelengths of $1 \mathrm{~h}$ have disappeared to a large extent while the long-wavelength portions become clearly visible. Slow sinusoidal, bump-like variations with amplitudes of about 0.1 and a half wavelength of $6 \mathrm{~h}$ (cf. component $\xi_{2 \mathrm{~h}}$, Fig. 4a, 18-24 h or Fig. 4d, 19-01 h) are apparent as well as wave-like structures with the same amplitude and $4 \mathrm{~h}$ duration (cf. Fig. $4 \mathrm{~b}$, component $\eta_{2 \mathrm{~h}}, 22-02 \mathrm{~h}$ or Fig. 4e, component $\xi, 18-22 \mathrm{~h}$ ). Moreover, also slowlyprogressing, drift-like fluctuations are visible, e.g. in Fig. 4c, component $\eta_{2 \mathrm{~h}}$. Contrary to $\eta$, the filtered components $\xi_{20 \mathrm{~m}}$ and $\xi_{2 \mathrm{~h}}$ generally tend to show positive differences in comparison with the reference value $\xi_{\text {ref }}$. Here, the systematic effect already mentioned above is visible. (cf. also Fig. 5.)

In order to further quantify the effects to be expected due to $\mathrm{AR}$, the differences between the filtered data series $(\xi, \eta)_{20 \mathrm{~m}}$ and $(\xi, \eta)_{2 \mathrm{~h}}$ and the reference value $(\xi, \eta)_{\text {ref }}$ may be analysed. The minimum and maximum differences are listed in Tables 7 and 8. For the 20-min-data, it can be seen that extreme values vary from night to night between 0. '1-0.'2 with maximum absolute values of up to 0.3 . The much smoother behaviour of the 2-hour-data is seen by the smaller absolute values varying between a few 0.01 and about 0.1 with a maximum below 0.22 . The general conclusion is that the mid- and low-frequency portion of AR with wavelengths of $2 \mathrm{~h}$ or more reaches amplitudes of the order of $0 . ' 1$ in zenith direction.

A problem of the filter technique applied is visible where data gaps occur (e.g. Fig. 4 e, component $\xi$, 00h-01h). Here, the amplitudes of the adjacent filtered data points are obviously falsified. The author is aware of this problem which could - at least partly - be mitigated by appropriate algorithms able to fill the gaps with synthetic data. However, as the largest part of the data sets is free of gaps, the main results are not affected. Moreover, it should not be unmentioned that adjacent data points of the filtered data sets are mathematically correlated due to the common processing (Eq. (2)). Larger random errors contained in the single observations may be responsible for a part of the amplitudes detected by means of $(\xi, \eta)_{20 \mathrm{~m}}$-data. However, due to the larger window width, this effect is considered to be negligible for the $(\xi, \eta)_{2 \mathrm{~h}}$-data.

\subsection{Accuracy assessment of filtered observations}

In order to estimate the accuracy of the filtered observation data, it is useful to compute the standard deviation with respect to comparison data. The accuracy numbers reflect both external influences (e.g. AR, star catalogue) and remaining, minor instrumental errors. It is easy to understand that the standard deviations computed with respect to the individual nightly mean values $(\xi, \eta)_{\text {ngt }}$ provide a too optimistic assessment of the obtained accuracy - as external systematic errors due to AR are not properly taken into account. Referring the standard deviations to the series mean $(\xi, \eta)_{\text {ser }}$ and especially to the highly-precise reference values $(\xi, \eta)_{\text {ref }}$ gives a more realistic estimation of the accuracy of vertical deflection data (Table 9). The accuracy of vertical deflection measurements $(\xi, \eta)_{20 \mathrm{~m}}$ is about $0{ }^{\prime} 07-0^{\prime \prime} 08$ whereas a 2h-observation is slightly better (0.'05-0.07), cf. Fig. 5. This estimation is in good agreement with previous results obtained with the same instrumentation (e.g. Hirt \& Seeber 2005). On the one hand, these figures underline the very low 

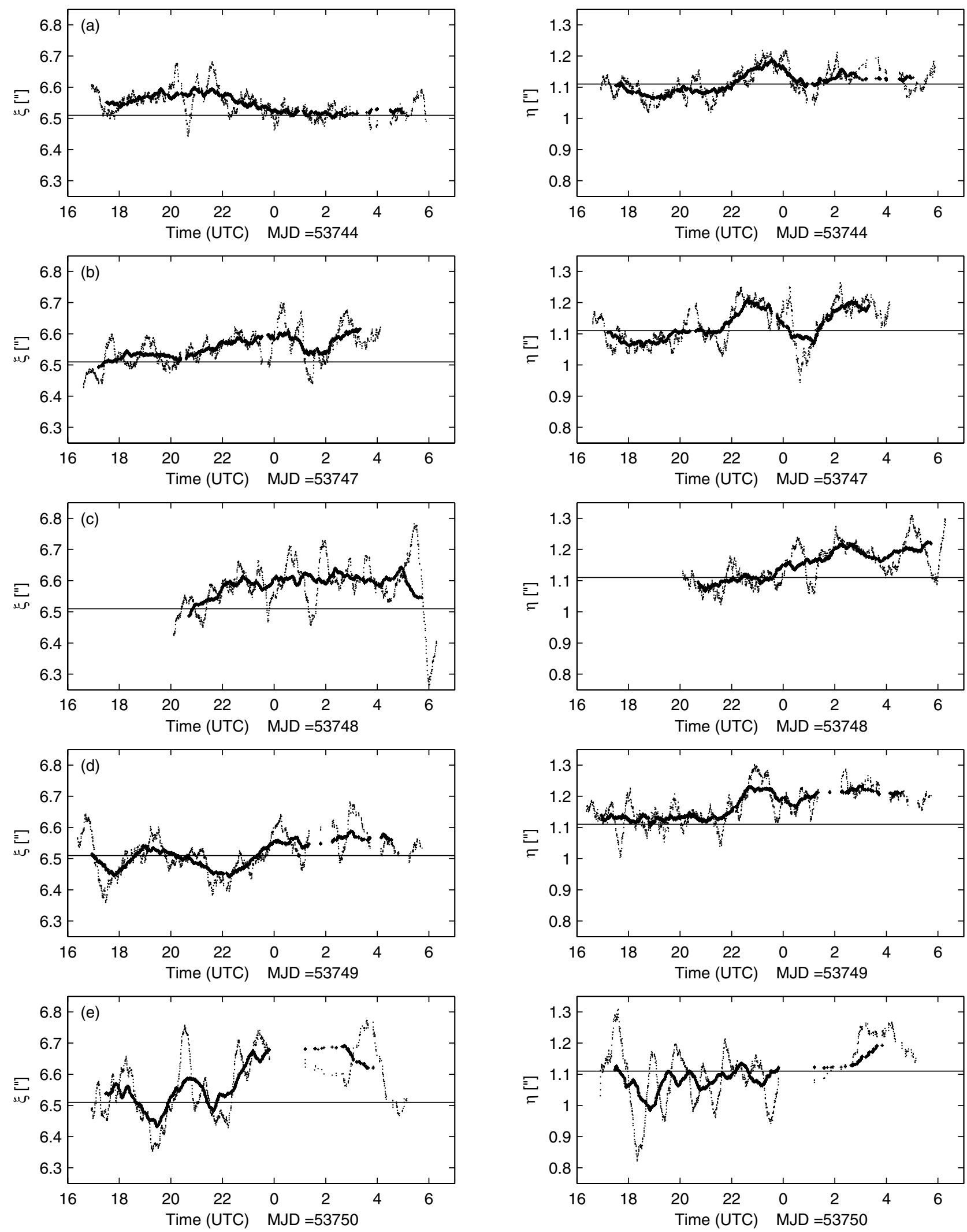

Fig. 4. Filtered observation data on 9th January and 12 th-15th January 2006 as a function of UTC. The 20-min-data $(\xi, \eta)_{20} \mathrm{~m}$ are shown with thin dots, the 2-hour-data $(\xi, \eta)_{2} \mathrm{~h}$ which are depicted with the + marker (appearing as thicker line) show a much smoother behaviour.

noise level of vertical deflection data sets $(\xi, \eta)$ acquired with the Digital Zenith Camera System TZK2-D. On the other hand the results presented so far make clear that the instrumentation is currently working near the limitations due to AR.

\subsection{Correlation analysis}

Analysing the cross-correlation between the meridian component $\xi$ and the component $\eta$ in the prime vertical may indicate 

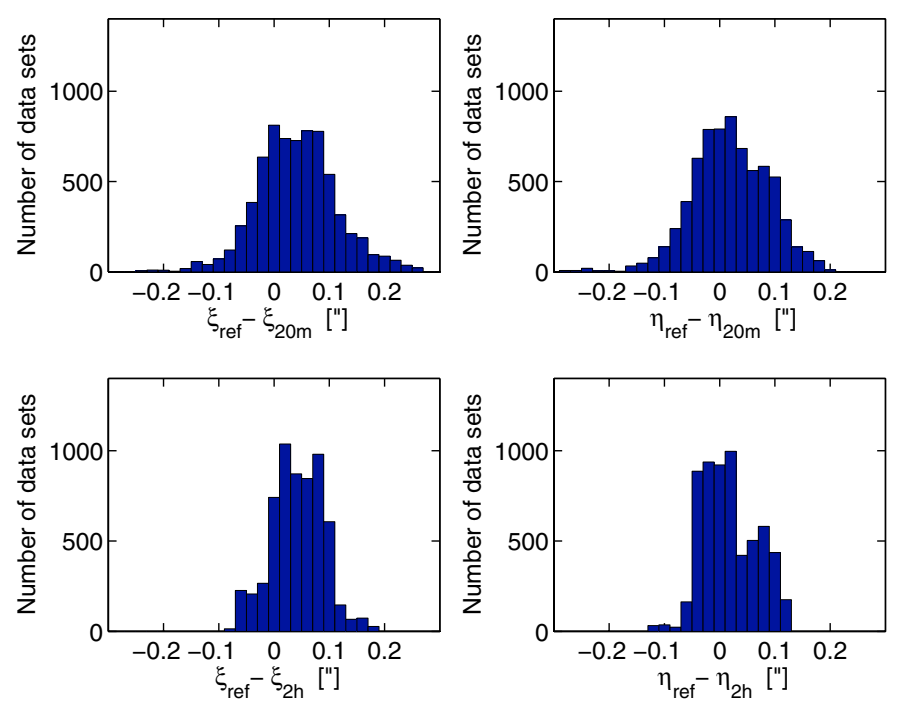

Fig. 5. Histogramm of the differences of filtered data sets $(\xi, \eta)_{20 \mathrm{~min}}$ (above) and $(\xi, \eta)_{2 \mathrm{~h}}$ (below) with respect to the reference values $(\xi, \eta)_{\text {ref }}$. The figure shows the high precision of the filtered data sets. The left subplots underline that a systematic effect is contained in the $\xi$ observations.

Table 7. Minimal and maximum differences of filtered data $(\xi, \eta)_{20 \mathrm{~m}}$ with respect to the highly-precise reference values $(\xi, \eta)_{\text {ref }}$.

\begin{tabular}{lcccc}
\hline \hline Date & \multicolumn{2}{c}{$\xi_{20 \mathrm{~m}}-\xi_{\text {ref }}$} & \multicolumn{2}{c}{$\eta_{20 \mathrm{~m}}-\eta_{\text {ref }}$} \\
& $\min \left[{ }^{\prime \prime}\right]$ & $\max \left[{ }^{\prime \prime}\right]$ & $\min \left[{ }^{\prime \prime}\right]$ & $\max \left[{ }^{\prime \prime}\right]$ \\
\hline 20060109 & -0.07 & 0.17 & -0.10 & 0.11 \\
20060110 & -0.08 & 0.14 & -0.12 & 0.04 \\
20060112 & -0.08 & 0.19 & -0.17 & 0.15 \\
20060113 & -0.24 & 0.27 & -0.09 & 0.20 \\
20060114 & -0.15 & 0.17 & -0.11 & 0.19 \\
20060115 & -0.15 & 0.27 & -0.29 & 0.19 \\
\hline Total & -0.24 & 0.27 & -0.29 & 0.20 \\
\hline
\end{tabular}

Table 8. Minimal and maximum differences of filtered data $(\xi, \eta)_{2 \mathrm{~h}}$ with respect to the highly-precise reference values $(\xi, \eta)_{\text {ref }}$.

\begin{tabular}{lcccc}
\hline \hline Date & \multicolumn{2}{c}{$\xi_{2 \mathrm{~h}}-\xi_{\text {ref }}$} & \multicolumn{2}{c}{$\eta_{2 \mathrm{~h}}-\eta_{\text {ref }}$} \\
& $\min \left[{ }^{\prime \prime}\right]$ & $\max \left[{ }^{\prime \prime}\right]$ & $\min \left[{ }^{\prime \prime}\right]$ & $\max \left[{ }^{\prime \prime}\right]$ \\
\hline 20060109 & -0.01 & 0.09 & -0.05 & 0.07 \\
20060110 & -0.01 & 0.11 & -0.07 & -0.03 \\
20060112 & -0.02 & 0.11 & -0.05 & 0.09 \\
20060113 & -0.02 & 0.14 & -0.05 & 0.11 \\
20060114 & -0.07 & 0.08 & -0.01 & 0.12 \\
20060115 & -0.08 & 0.18 & -0.13 & 0.08 \\
\hline Total & -0.08 & 0.18 & -0.13 & 0.12 \\
\hline
\end{tabular}

whether externally caused effects are contained in the data. The correlation analysis assumes that an external error source like AR is likely to affect both $\xi$ and $\eta$ with the result that the residual differences (with respect to mean values) are statistically dependent. The cross-correlation between $\xi$ and $\eta$ approaches 0 when random errors prevail and also in those cases when the maximum tilt of atmospheric layers and hence amplitude of AR exclusively occurs in the meridian direction (azimuth of $0^{\circ}$ or $180^{\circ}$ ) or in the direction of the prime vertical (azimuth of $90^{\circ}$ or $270^{\circ}$ ). A strong positive correlation coefficient near 1 indicates similarly affected components $\xi$ and $\eta$, corresponding to a maximum tilt of atmospheric layers in North-East or South-West direction
Table 9. Standard deviation of the filtered observation data $(\xi, \eta)_{20 \mathrm{~m}}$ and $(\xi, \eta)_{2 \mathrm{~h}}$ with respect to their nightly mean values $(\xi, \eta)_{\text {ngt }}$ and the highly-precise reference values $(\xi, \eta)_{\text {ref }}$.

\begin{tabular}{lcccc}
\hline \hline Reference & $s_{\xi 20 \mathrm{~m}}\left[^{\prime \prime}\right]$ & $s_{\eta 20 \mathrm{~m}}\left[^{\prime \prime}\right]$ & $s_{\xi 2 \mathrm{~h}}\left[{ }^{\prime \prime}\right]$ & $s_{\eta 2 \mathrm{~h}}\left[^{\prime \prime}\right]$ \\
\hline$(\xi, \eta)_{\text {ngt }}$ & 0.067 & 0.061 & 0.040 & 0.035 \\
$(\xi, \eta)_{\text {ser }}$ & 0.070 & 0.069 & 0.046 & 0.051 \\
$(\xi, \eta)_{\text {ref }}$ & 0.083 & 0.069 & 0.065 & 0.049 \\
\hline
\end{tabular}

Table 10. Cross-correlation coefficients between $\xi$ and $\eta$. The bottom line gives the mean absolute cross-correlation coefficients.

\begin{tabular}{lccc}
\hline \hline Date & $\begin{array}{c}\text { Single } \\
\text { Observation }\end{array}$ & $\begin{array}{c}20 \text { min- } \\
\text { window }\end{array}$ & $\begin{array}{c}2 \mathrm{~h}- \\
\text { window }\end{array}$ \\
\hline 20060109 & -0.03 & -0.42 & -0.61 \\
20060110 & +0.01 & -0.30 & -0.47 \\
20060112 & +0.13 & +0.32 & +0.62 \\
20060113 & +0.03 & +0.32 & +0.61 \\
20060114 & +0.08 & +0.26 & +0.45 \\
20060115 & -0.08 & -0.11 & +0.45 \\
\hline Mean abs. & 0.06 & 0.29 & 0.54 \\
\hline
\end{tabular}

(azimuth of $45^{\circ}$ or $225^{\circ}$, respectively). A clear negative correlation may be expected for azimuths near $135^{\circ}$ and $305^{\circ}$. On the other hand, mixed cases (presence of random errors and intermediate azimuths) lead to a weaker correlation.

The cross-correlation coefficients have been computed for single observations $\xi$ and $\eta$, and for the filtered data classes $(\xi, \eta)_{20 \mathrm{~m}}$ and $(\xi, \eta)_{2 \mathrm{~h}}$, respectively (Table 10$)$. The correlation between single observations is nearly zero as the random error sources are clearly dominating. A considerable cross-correlation exists between the components $\xi$ and $\eta$ of the filtered 2-hourdata. While the coefficients are negative for the first two nights $(-0.61$ and -0.47$)$, they range between +0.45 and +0.62 for the remaining data sets. The coefficients are considered to give a good indication for a correlating AR effect which is occurring in different spatial directions in different nights as revealed by the smoothed 2-hour-data.

A weaker cross-correlation of about 0.3 (and -0.3 for the first nights) has been found for the filtered 20-min-data as such indicating a slight correlation. Consequently, a larger portion of random errors is still contained in the 20-min-data. Therefore, the wave-like fluctuations of the 20-min-data which were discussed in the previous section have to be treated with caution as other, more randomly appearing effects may be responsible for at least a smaller part of the amplitudes.

\section{Discussion and outlook}

The time series analysis of the filtered 2-hour-data $(\xi, \eta)_{2 \mathrm{~h}}$ reveals amplitudes of about 0 .' 1 occurring as bump-like, wave-like or slowly fluctuating pattern with wavelengths of $4 \mathrm{~h}, 6 \mathrm{~h}$, or even longer. The 20-min-data show - in essence - a various number of wave-like structures with amplitudes of about $0.1-0.2$. The correlation analysis indicates a moderate cross-correlation for the 20-min-data and a considerable correlation between the components of the 2-hour-data. This supports the assumption that at least the amplitudes appearing in the 2-hour-data are induced by an external effect.

Instrumental effects can be excluded to be the cause for the wave-like structures because instrumental tilt and variations of sensor offsets are compensated by modelling and sophisticated 
observation strategies. External influences basically may originate from the star catalogue and from the AR field. A possible systematic, wave-like effect coming from the star catalogue UCAC would show similar features in consecutive nights with a time shift of about 4 min as the same star fields are used. This is obviously not the case.

Therefore, the major part of the structures shown in Figs. 3 and 4 is believed to originate from AR. The order of amplitudes is in accordance with those of previous studies mentioned in the first section. It can be assumed that the heterogeneous environment of the observation site as initially described promotes the occurrence of local, fluctuating anomalous refraction effects.

Even an extremely long wavelength effect appears in the meridian component $\xi$. The associated systematic deviation is of about 0. '04.

It seems reasonable to assume that this effect is unlikely to be mitigated by additional observations carried out in the same season. The cause for this slight systematic effect is currently not clear. It can be explained as a season-dependent heat island effect appearing locally due to the location of the observation site. Another possible source of this deviation could be a seasondependent, more regionally occurring tilt of atmospheric layers.

The presented results are based on observations carried out in zenith direction. In other directions (e.g. zenith angles of $30^{\circ}$ ), AR multiplies as derived by e.g. Bretterbauer (1965); Ramsayer (1970). Therefore this study indicates the minimum of AR to be expected when absolute astrometric observations are carried out.

A next step aiming at a further confirmation of the presented characteristics of AR could be to monitor the refraction field with two Digital Zenith Camera Systems in parallel operation. $\mathrm{AR}$, similar to that indicated in this study, may be expected to influence both sets of observation data in a similar way thus leading to a significant correlation between both data sets.

A further important outcome of the study is the considerable accuracy of the Digital Zenith Camera System TZK2-D. The accuracy of the filtered data was estimated with respect to highly-precise reference values available at the observation site. The accuracy of the mean values computed from a 20-min observation has been found to be about 0 . $^{\prime} 07-00^{\prime} 08$. The one of $2-\mathrm{h}$ observation is slightly better with about 0 ' $^{\prime} 05-0$ ' $^{\prime} 07$. The accuracy numbers demonstrate that the mobile measurement system TZK2-D is currently doing absolute astrometry in the vicinity of the limitations coming from anomalous refraction.

Acknowledgements. The study was supported by German National Research Foundation DFG. The author is grateful to the students Rene Gudat, Niels
Hartmann, Tobias Krömer, Eiko Münstedt and Ilka Rehr who operated the instrument during the long cold winter nights in January 2006. Carl Christian Tscherning (Copenhagen) is kindly acknowledged for his early idea to perform continuous observations with the new generation of Digital Zenith Cameras. The author would like to thank Norbert Zacharias (Washington) and his team for the high-precision UCAC reference data enabling to study anomalous refraction. Lars Winter (Hamburg), Günter Seeber (Hannover) and an unknown referee are kindly acknowledged for their comments on the manuscript.

\section{References}

Auer, L. H., \& Standish, E. M. 2000, AJ, 119, 2472

Bretterbauer, K. 1965, Österreichische Zeitschrift für Vermessungswesen 53, Nr. 4,113

Christian, C., \& Racine, R. 1985, PASP, 97, 1215

Currie, D. G. 1979, in IAU Symposium Refractional Influences in Astrometry and Geodesy, ed. E. Tengström \& G. Teleki, 131

Dimopoulos, T. 1982, Ph.D. Thesis, Fakultät Bauingenieur- und Vermessungswesen der Universität Stuttgart

Han, I., \& Gatewood, G. D. 1995, PASP, 107, 399

Hirt, C. 2003, ASP Conf. Ser., ed. H. E. Payne, et al., 295, 156

Hirt, C. 2004, Ph.D. Thesis, Wissenschaftliche Arbeiten der Fachrichtung Geodäsie und Geoinformatik an der Universität Hannover Nr., 253. URL: http://edok01.tib.uni-hannover.de/edoks/ eQ1dh04/393223965.pdf

Hirt, C., \& Bürki, B. 2002, Proceed. 3rd Meeting Intern, Gravity and Geoid Commission of the IAG, Thessaloniki, ed. I. Tziavos, 161

Hirt, C., \& Seeber, G. 2005, High-Resolution Local Gravity Field Determination at the Sub-Millimeter Level using a Digital Zenith Camera System, Proceedings IAG Meeting "Dynamic Planet", Cairns 2005, in press

Høg, E. 1968, ZAp, 69, 313

Hughes, J. A. 1979, In IAU Symposium Refractional Influences in Astrometry and Geodesy, ed. E. Tengström, \& G. Teleki, 13

Kovalevsky, J. 1998, ASP Conf. Ser., ed. Isobe, S. \& T. Hirayama, 139, 89

Nakajima, K. 1979, PASJ, 31, 775

Olmi, L. 2001, A\&A, 374, 348

Pier, J. R., Munn, J. A., Hindsley, R. B., et al. 2003, AJ, 125, 1559

Ramsayer, K. 1967, Österreichische Zeitschrift für Vermessungswesen, Sonderheft 25, 260

Ramsayer, K. 1970, Handbuch der Vermessungskunde Band IIa - Geodätische Astronomie, Metzlersche Verlagsbuchhandlung, Stuttgart

Schubart, J. 1954, Astron. Nachr., 281, 255

Stone, R. C. 1996, PASP, 108, 1051

Stone, R. C., Monet, D. G., Monet, A. K. B., et al. 1996, AJ, 111, 1721

Sugawa, C. 1956, PASJ, 8(1), 27

Sugawa, C. 1958, PASJ, 10(2), 79

Sugawa, C., \& Kikuchi, N. 1979, In IAU Symposium Refractional Influences in Astrometry and Geodesy, ed. E. Tengström \& G. Teleki, 103

Teleki, G. 1979, In IAU Symposium Refractional Influences in Astrometry and Geodesy, ed. E. Tengström \& G. Teleki, 1

Vondrák, J., Ron, C., Pesek, I., \& Cepek, A. 1995, A\&A, 297, 899

Wittmann, A. D. 1997, Astron. Nachr., 318, 305

Zacharias, N., Urban, S. E., Zacharias, M. I., et al. 2004, AJ, 127, 3043 